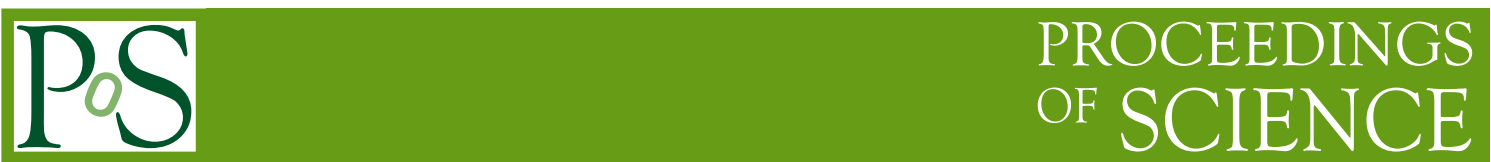

\title{
Study of b-hadron properties with semileptonic decays
}

\author{
Florian Reiss ${ }^{\dagger, *}$ \\ LPNHE, Sorbonne Université, Paris Diderot Sorbonne Paris Cité, CNRS/IN2P3, Paris, France \\ E-mail: florian.reiss@cern.ch
}

With large branching fractions and controllable theoretical uncertainties, semileptonic $B$ decays are excellent tools for measuring $b$-hadron properties as well as testing QCD calculations. The large samples of $B_{s}^{0}$ mesons and $b$-baryons uniquely available at LHCb extend the experimental reach into this sector, allowing differential decay rates of these hadrons to be probed for the first time. The observation and study of $B^{+} \rightarrow p \bar{p} \mu^{+} v_{\mu}$ decays offer the opportunity to investigate hadronic meson-to-baryon-pair transitions. The most recent measurements of $b$-hadron properties using semileptonic $b$-hadron decays are presented.

40th International Conference on High Energy physics - ICHEP2020

July 28 - August 6, 2020

Prague, Czech Republic (virtual meeting)

\footnotetext{
*Speaker

$\dagger$ on behalf of the LHCb collaboration
} 


\section{Introduction}

The LHCb detector $[1,2]$ is an ideal testing ground for studying the properties of $b$-hadrons. Its trigger system is designed for the displaced signature of their decays and a large amount of $b$ hadrons are produced, including $B_{s}^{0}, B_{c}^{+}$and $\Lambda_{b}^{0}$ hadrons ${ }^{1}$. Studying them using semileptonic decays takes advantage of their generally large branching fractions. Also, the uncertainties on theoretical predictions are well understood as the transition involves only one hadronic current, which can be parametrised by form factors. In the following, measurements performed using (parts of the) data collected by LHCb at centre-of-mass energies $\sqrt{s}$ of 7,8 and $13 \mathrm{TeV}$ are summarized.

\section{Measurement of b-hadron fractions in $13 \mathrm{TeV} p p$ collisions}

The $B_{s}^{0}$ and $\Lambda_{b}^{0}$ hadron production fractions are measured relative to the sum of the $B^{-}$and $\bar{B}^{0}$ meson production fractions using data corresponding to an integrated luminosity of $1.67 \mathrm{fb}^{-1}$ recorded at $13 \mathrm{TeV}$ in 2016 [3]. This measurement extends the previous measurement of the same quantities performed at $\sqrt{s}=7 \mathrm{TeV}$ [4]. Knowledge of the relative production fractions is a requirement for measuring absolute branching fractions of decays of these hadrons. The measurement is performed in bins of the transverse momentum $p_{\mathrm{T}}$ and the pseudorapidity $\eta$ of the hadron. The $b$-hadron yields are determined using inclusive $H_{b} \rightarrow H_{c} \mu v\left(H_{c}=D_{s}^{+}, \Lambda_{c}^{+}, D^{0}, D^{+}\right)$ decays. The yields are then corrected by the corresponding branching fractions and selection efficiencies. The measured relative production fraction as a function of the transverse momentum of the hadron is shown in Fig. 1, where a strong dependence on the transverse momentum is seen for the $\Lambda_{b}^{0}$ hadron and a light dependence for the $B_{s}^{0}$ hadron. No dependence on the pseudorapidity is observed. Integrating over the fiducial volume of $4<p_{\mathrm{T}}<25 \mathrm{GeV}, 2<\eta<5$, the fractions are

$$
\begin{aligned}
& \frac{f_{s}}{f_{u}+f_{d}}=0.122 \pm 0.006(\text { stat } .+ \text { syst } .), \\
& \frac{f_{\Lambda_{b}^{0}}}{f_{u}+f_{d}}=0.259 \pm 0.018(\text { stat } .+ \text { syst } .),
\end{aligned}
$$

where the statistical and systematic uncertainties are combined.

\section{Measurement of the $B_{c}^{-}$meson production fraction in 7 and $13 \mathrm{TeV} p p$ collisions}

Similar to the previous section, the $B_{c}^{-}$meson production fraction is measured using data collected at centre-of-mass energies of 7 and $13 \mathrm{TeV}$ [5]. The $B_{c}^{-}$meson is reconstructed in the $B_{c}^{-} \rightarrow J / \psi \mu^{-} \bar{v}_{\mu}$ decay. Its yield is determined by fitting the corrected mass $m_{c o r r}$ defined as

$$
m_{\text {corr }}=\sqrt{m_{J / \psi \mu^{-}}^{2}+p_{\perp}^{2}}+p_{\perp},
$$

where $p_{\perp}$ is the magnitude of the $\left(J / \psi \mu^{-}\right)$momentum component transverse to the flight direction of the $B_{c}^{-}$meson. The measurement relies on the theoretical prediction of $\mathcal{B}\left(B_{c}^{-} \rightarrow J / \psi \mu^{-} \bar{v}_{\mu}\right)$. The average value of the theory predictions of $\mathcal{B}\left(B_{c}^{-} \rightarrow J / \psi \mu^{-} \bar{v}_{\mu}\right)=1.95 \% \pm 0.46 \%$ is used.

\footnotetext{
${ }^{1}$ charge conjugation is implied throughout this document
} 


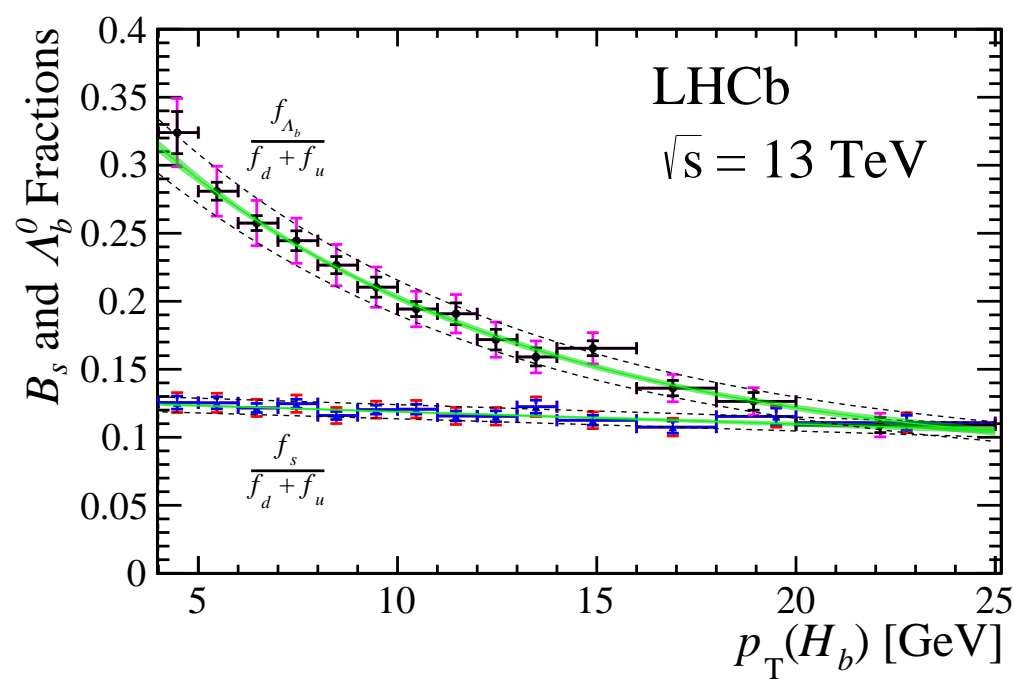

Figure 1: The ratios $f_{s} /\left(f_{u}+f_{d}\right)$ and $f_{\Lambda_{b}^{0}} /\left(f_{u}+f_{d}\right)$ in bins of $p_{\mathrm{T}}\left(H_{b}\right)$ [3]

No strong dependence on $\eta$ is found for the relative $B_{c}^{-}$production fraction, but a decrease of the production fraction with the transverse momentum of the $B_{c}^{-}$hadron is observed, as shown in Fig. 2 The average relative production fraction in the fiducial volume is determined to be

$$
\begin{aligned}
& \frac{f_{c}}{f_{u}+f_{d}}=(3.63 \pm 0.08(\text { stat. }) \pm 0.12(\text { syst. }) \pm 0.86(\text { ext. })) \cdot 10^{-3} \text { for } 7 \mathrm{TeV}, \\
& \frac{f_{c}}{f_{u}+f_{d}}=(3.78 \pm 0.04(\text { stat. }) \pm 0.15(\text { syst } .) \pm 0.89(\text { ext. })) \cdot 10^{-3} \text { for } 13 \mathrm{TeV}
\end{aligned}
$$

where the first uncertainty is statistical, the second systematic and the third due to the uncertainty on the prediction of $\mathcal{B}\left(B_{c}^{-} \rightarrow J / \psi \mu^{-} \bar{v}_{\mu}\right)$.
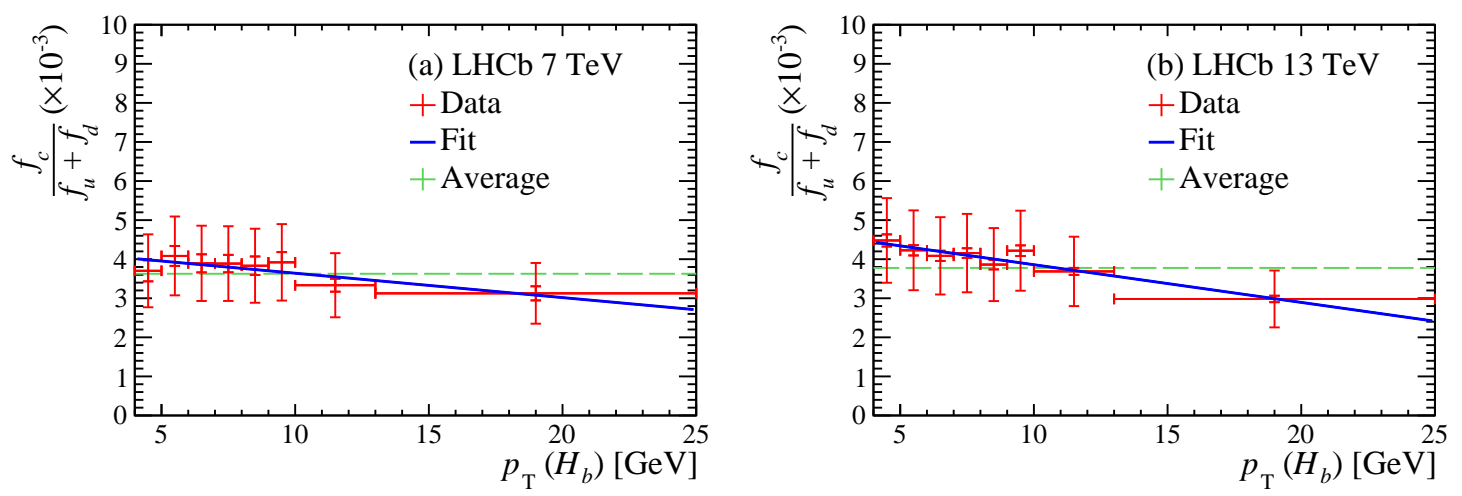

Figure 2: Ratio $f_{c} /\left(f_{u}+f_{d}\right)$ as a function of $p_{\mathrm{T}}\left(H_{b}\right)$ in $7 \mathrm{TeV}$ (left) and $13 \mathrm{TeV}$ (right) data [5].

\section{Observation of the semileptonic decay $B^{+} \rightarrow p \bar{p} \mu^{+} v_{\mu}$}

The Belle collaboration reported evidence for the $B^{+} \rightarrow p \bar{p} \mu^{+} v_{\mu}$ decay [6]. The first observation of this decay is made using data recorded at $\mathrm{LHCb}$ corresponding to integrated luminosities 
of $1 \mathrm{fb}^{-1}, 2 \mathrm{fb}^{-1}$ and $1.7 \mathrm{fb}^{-1}$ at centre-of-mass energies of 7,8 and $13 \mathrm{TeV}$ respectively [7]. The signal yield is extracted by fitting the corrected mass

$$
m_{c o r r}=\sqrt{m_{p \bar{p} \mu}^{2}+p_{\perp}^{2}}+p_{\perp}
$$

in bins of the $p \bar{p}$ invariant mass and the corresponding branching fraction is measured relative to the $B^{+} \rightarrow J / \psi\left(\rightarrow \mu^{+} \mu^{-}\right) K^{+}$branching fraction.

The overall branching fraction is determined to be

$$
\mathcal{B}\left(B^{+} \rightarrow p \bar{p} \mu^{+} v_{\mu}\right)=\left(5.27_{-0.24}^{+0.23}(\text { stat. }) \pm 0.21(\text { syst. }) \pm 0.15(\text { ext. })\right) \times 10^{-6},
$$

where the first uncertainty is statistical, the second systematic and the third due to the uncertainty on the $B^{+} \rightarrow J / \psi\left(\rightarrow \mu^{+} \mu^{-}\right) K^{+}$branching fraction. The measured value is found to be lower than the theory prediction from perturbative QCD [8]. The measured branching fraction in bins of the $p \bar{p}$ invariant mass is shown in Fig. 3. Also shown is the theoretical prediction scaled to the measured value. The observed shape of the differential branching fraction is consistent with the theory expectation and an enhancement of the branching fraction at the $p \bar{p}$ threshold is seen.

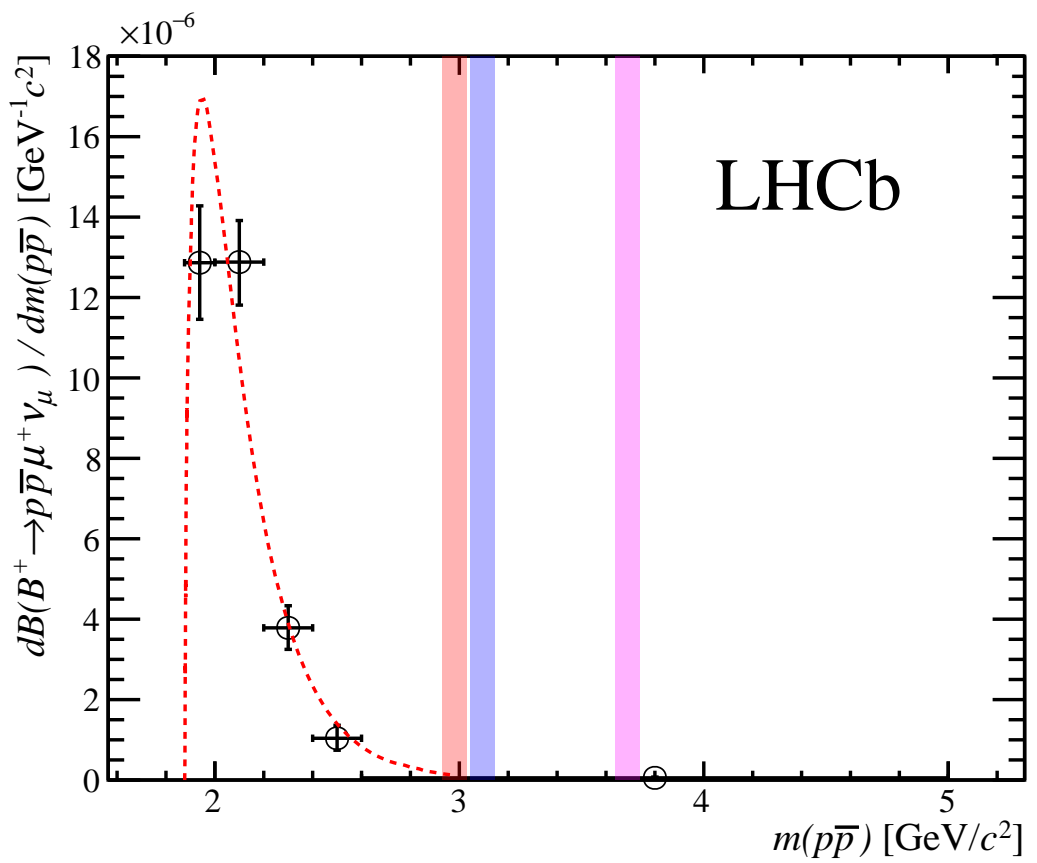

Figure 3: Differential branching fraction of the $B^{+} \rightarrow p \bar{p} \mu^{+} v_{\mu}$ decay as a function of the $p \bar{p}$ invariant mass. The red dashed line shows the prediction from perturbative QCD normalised to the observed branching fraction. The $\eta_{c} \rightarrow p \bar{p}, J / \psi \rightarrow p \bar{p}$ and $\psi(2 S) \rightarrow p \bar{p}$ regions, which are vetoed in the measurement, are shown as red, blue and pink bands, respectively [7].

\section{Measurement of the shape of the $B_{s}^{0} \rightarrow D_{s}^{*-} \mu^{+} v_{\mu}$ differential decay rate}

The shape of the differential decay rate of the $B_{s}^{0} \rightarrow D_{s}^{*-} \mu^{+} v_{\mu}$ decay is measured using data corresponding to an integrated luminosity of $1.7 \mathrm{fb}^{-1}$ at a centre-of-mass energy of $13 \mathrm{TeV}$ [9]. The 
$D_{s}^{*-}$ is reconstructed in the $D_{s}^{*-} \rightarrow D_{s}^{-} \gamma$ final state and the $D_{s}^{-}$through the $D_{s}^{-} \rightarrow K^{-} K^{+} \pi^{-}$decay. The signal yield is extracted by fitting the corrected mass distribution

$$
m_{\text {corr }}=\sqrt{m_{D_{s}^{*-} \mu^{+}}^{2}+p_{\perp}^{2}}+p_{\perp}
$$

in bins of the hadron recoil $w$

$$
w=\frac{p_{B_{s}^{0}}}{m_{B_{s}^{0}}} \frac{p_{D_{s}^{*-}}}{m_{D_{s}^{*-}}}=\frac{m_{B_{s}^{0}}^{2}+m_{D_{s}^{*-}}^{2}-q^{2}}{2 m_{B_{s}^{0}} m_{D_{s}^{*-}}},
$$

where $q^{2}$ is the squared momentum transfer to the lepton system $q^{2}=\left(p_{B_{s}^{0}}-p_{D_{s}^{*-}}\right)^{2}$. The obtained distribution of $w$ is unfolded to account for the resolution of $w$ and corrected by the efficiency. The resulting $w_{\text {unf }}$ distribution is fitted using the CLN [10] and BGL [11] form factor parametrisations, as shown in Fig. 4. The two form factor models are observed to be consistent with each other and the data. This is the first time the normalised differential decay rate of the $B_{s}^{0} \rightarrow D_{s}^{*-} \mu^{+} v_{\mu}$ decay is measured.

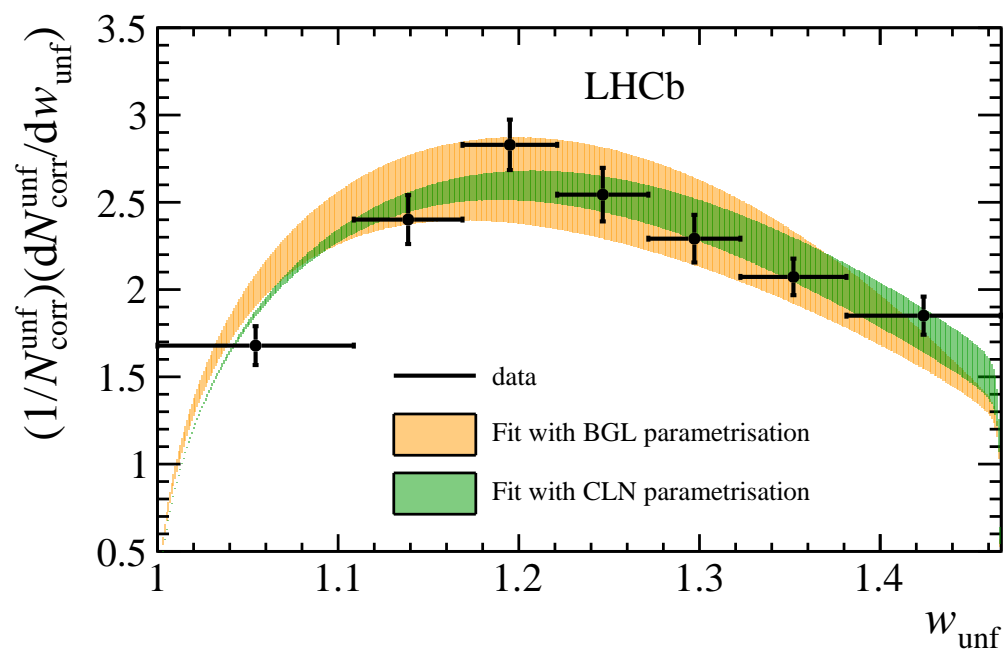

Figure 4: Unfolded normalised differential $B_{s}^{0} \rightarrow D_{s}^{*-} \mu^{+} v_{\mu}$ decay rate with the fit results for the CLN and BGL parametrisations [9].

\section{Conclusions}

Using the large data sample it has recorded, the LHCb collaboration studied various $b$-hadron properties. Particularly, the relative production fractions of $B_{s}^{0}, \Lambda_{b}^{0}$ and $B_{c}^{-}$hadrons are measured. Investigation of the data sample has also led to the first observation of the Cabibbo-suppressed $B^{+} \rightarrow p \bar{p} \mu^{+} v_{\mu}$ decay and the first-time measurement of the normalised differential decay rate of the $B_{s}^{0} \rightarrow D_{s}^{*-} \mu^{+} v_{\mu}$ decay. Measuring these properties provides important input for further measurements and theory predictions. The relative production fractions are required to extract absolute branching fractions. Experimental determination of form factors is a necessary input to predict relative branching fractions used in lepton universality tests. Furthermore, predictions by (perturbative) QCD can be explicitly tested. 


\section{Acknowledgments}

FR acknowledges funding from the European Research Council (ERC) under the European Union's Horizon 2020 research and innovation programme under grant agreement No 724777 "RECEPT".

\section{References}

[1] LHCв collaboration, The LHCb detector at the LHC, JINST 3 (2008) S08005.

[2] LHCв collaboration, LHCb detector performance, Int. J. Mod. Phys. A30 (2015) 1530022 LHCB-DP-2014-002, CERN-PH-EP-2014-290, [1412 . 6352].

[3] LHCв collaboration, Measurement of b hadron fractions in $13 \mathrm{TeV}$ pp collisions, Phys. Rev. D 100 (2019) 031102 [1902.06794].

[4] LHCв collaboration, Measurement of b hadron production fractions in $7 \mathrm{TeV} p p$ collisions, Phys. Rev. D 85 (2012) 032008.

[5] LHCв collaboration, Measurement of the $B_{c}^{-}$meson production fraction and asymmetry in 7 and $13 \mathrm{TeV}$ pp collisions, Phys. Rev. D 100 (2019) 112006.

[6] Belle collaboration, Evidence for semileptonic $B^{-} \rightarrow p \bar{p} \ell^{-} \bar{v}_{\ell}$ decays, Phys. Rev. D 89 (2014) 011101.

[7] LHCв collaboration, Observation of the semileptonic decay $B^{+} \rightarrow p \bar{p} \mu^{+} v_{\mu}$, JHEP 2003 (2019) 146. 23 p.

[8] C. Geng and Y. Hsiao, Semileptonic $B^{-} \rightarrow p \bar{p} \ell^{-} \bar{v}_{\ell}$ decays, Phys. Lett. B 704 (2011) 495 [1107.0801].

[9] LHCв collaboration, Measurement of the shape of the $B_{s}^{0} \rightarrow D_{s}^{*-} \mu^{+} v_{\mu}$ differential decay rate, JHEP 12 (2020) 144 [2003.08453].

[10] I. Caprini, L. Lellouch and M. Neubert, Dispersive bounds on the shape of $\bar{B} \rightarrow D^{(*)} \ell \bar{v}$ form-factors, Nucl. Phys. B 530 (1998) 153 [hep-ph/9712417].

[11] C. Boyd, B. Grinstein and R.F. Lebed, Model independent extraction of $|V(c b)|$ using dispersion relations, Phys. Lett. B 353 (1995) 306 [hep-ph/9504235]. 